\title{
XXX. On the method of extracting iodine from Kelp
}

\section{Van Mons}

To cite this article: M. Van Mons (1820) XXX. On the method of extracting iodine from Kelp, Philosophical Magazine Series 1, 55:263, 207-213, DOI: $10.1080 / 14786442008652300$

To link to this article: http://dx.doi.org/10.1080/14786442008652300

曲 Published online: 23 Jul 2009.

Submit your article to this journal $\widetilde{ }$

III Article views: 4

Q View related articles $₫$ 
from what has been already said, and may be thus summed up in few words : viz. To encourage and promote their peculiar science by every means in their power, but especially - by collecting, reducing, and publishing useful observations and tables-by setting on foot a minute and systematic examination of the heavens- by encouraging a general spirit of inquiry in practical astronomyby establishing communications with foreign observers-by circulating notices of all remarkable phrenomena about to bappen, and of discoveries as they arise-by comparing the merits of dif ferent artists eminent in the construetion of astronomical instruments-by proposing prizès for the improvement of particular departments, and bestowing medals or rewards on suecessful research in all-and finally, by acting as far as possible, in concert with every institution, both in England and abroad, whose objects have any thing in common with their own; but avoiding all interference with the objeets and interests of established scientific bodies.

XXX. On the Method of extracting Iodine from Kelp. By $M$. VAN MONs*.

$\mathbf{T}$ Hosk chemists who have directed their attention to a method for obtaining iodine by the decomposition of the iodate of potash existing in kelp, have proposed several methods for the purpose. Some recommend to separate from the lye of the kelp all the crystallizable salts, and to treat the mother-water with sulphuric acid;-others advise that the sub-carbonate of soda alone should be separated, and the liquid or the remaining salts treated with sulphuric acid; - a third class suggest methods more or less complicated, among which they cite as advantageous the addition of the superoxide of manganese, or the hypo-superoxide of lead; -and lastly, it has been prescribed to separate the iodate from the other salts, by taking advantage of its solubility in alcohol.

Each of these processes has its inconveniences. In adopting the first, there is a risk of making the sulphuric acid act on the mother water, which often contains no more than one atom of salt to furnish the iodine. We have experienced this, and have remarked, that from the moment that the greater part of the salts became separated by crystallization, and that the lye began to thicken, when it was desired to carry the separation further, the salt containing the iodic acid was at the sume time carried away; already even more or less of it had passed with the first crystals ; so that, when the mother-water contained no more crystallizable salts, there ordinarily remained no more iodate.

* From Annales Gererales des Scinces Physiqucs. 
The second process is attended with the inconvenience of subjecting a large mass of salt to the decomposing action of sulphuric acid, before the latter can begin to operate upon the iodate; for it is not till after the other salts, and especially the muriates, are decomposed, that an excess of sulphuric acid attacks the iodate of potash, and separates from it the alkali, which at the same instant oxygenizes itself at the expense of a portion of the same acid. If in place of muriates the solution contains nitrates, the decomposition of the latter suffices to oxygenate the iodic acid, on account of the facility with which the nitric acid susceptible of suboxygenation gives forth its oxygen. By extracting from the lye nothing but the sub-carbonate of soda, we obtain what is of little or no use; for the different sodas of Normandy with which we have made experiments, do not even contain as much of this salt as will produce a change in the tincture of curcuma. How, besides, can it. be conceived that there exists at the same time in a lye a free alkali and deliquescent muriates? The express condition that the muriates shall be deeomposed previous to the iodate, occasions an excessive expenditure of acid, besides the inconvenience of prolonging the operation, and atlowing much iodine to escape, if the precaution is neglected of not allowing the matter to get into ebullition before the greater part of the muriatic acid is expelled.

The process in which they mingle the superoxide of manganese with the residue is scarcely any better; it occasions a production of chlorine which contains the iodine in combination. The addition of the superoxide may be more or less permitted, where iodate exempt from muriate is made use of ; the superoxide may then serve rather to regenerate the sulphuric acid by the oxygenation of the sulphurous acid, than to oxygenate the iodic acid. This addition, taking care not to add at once all the sulphuric acid, may further have the effect of facilitating the separation of the muriatic acid, by converting it into chlorine; but the presence of chlorine produces such a disposition to the formation of iodine, and the affinity between the two bodies is so manifest, that a very great loss of iodine must be expected.

We shall not say any thing of the process by which the iodate of potash is isolated by means of alcohol: it requires that the muriates of lime and magnesia contained in the lye should be previously decomposed by the subcarbonate of potash; otherwise these muriates, being soluble in alcohol, would become confounded with the iodine. Besides, this process can only be employed in demonstrations.

Our results are infinitely more advantageous. We reduce the rough kelp into powder, and pass it through a hair-sieve; when it is not dry enough to be subnitted to that pulverization, we 
pound it well in a mortar with water, and make it boil for half an hour, stirring it continually with an iron spatula; we then filter the mixture and set it to evaporate, taking care to lift up with a skimmer the salts, a certain quantity of which the hot lye allows to be deposited. If evaporated till crystalization takes place by cooling, the salts become confounded in the crystallization, and a great deal of iodate remains adhering to the crystals.

The crystals, however weak may have been the lye in which they are formed, are always impregnated with iodate, which is in a state of such strong combination, that these crystals, although perfectly dried, redden strongly with sulphuric acid. It is better, therefore, to allow the salt to be deposited in the hot lye, where the muriates are not much more soluble than in the cold lye, where the iodate remains a much longer time in solution. The salt on being separated from the lye, and while it is still hot, should be spread on a hair-sieve, where it may dry itself. When about half of the salt has been dried, it is put aside in order to be afterwards pulverised and washed. The evaporation is continued with the same precaution-that is to say, removing the salt in proportion as the crystals are formed, until it ceases to form any more. The lye is then united with the drain water, and evaporated to dryness.' A single lixiviation will then suffice to exhaust the kelp of its salt.

We would advise that this salt should not be thrown away, as there still adheres to it a good deal of iodate, until it has been passed through a hot funnel. As the first crystallizations of this salt redden with sulphuric acid as much as the last, they equally contain iodate, which it is necessary to separate. For this purpose the salt is well shaken in large glass fumnels; and by little and little warm water is passed though it, trying at each washing whether the salt continues to redden with sulphuric acid.

The residue of the lye evaporated to dryness gets soon damp in the air. It is necessary, therefore, to preserve it in flasks well corked, if the iodine is not immediately wanted: when the latter is the case, the residue is put into an earthen pan, and pounded. continually, incorporating with it by instillation concentrated sul.phuric acid; we say by instillation; for if a drop of acid rests on the place where it falls, a spot of iodine is soon manifested, and a degree of heat developed which it is of importance to avoid.

When enough of acid has been added to decompose the muriate and the iodate, the mixture is transferred to a glass or earthen alembic in order to be distilled. 'The heat at first being moderate, ought not to be carried to that degree of ebullition that no more muriatic acid is seen to pass, otherwise the iodine, which at that temperature instantly evaporates, may be in part dissolved by that acid.

Vol. 55. No. 263. March 1820. 


\section{On the Method of extracting Iodine from Kelp.}

After iodine has ceased to be disengaged, in order to try if the residue is exhausted, a new quantity of sulphuric acid is poured upon it while it is still hot; if at the moment of contact a white vapour without any tinge of red arises, it is a proof that the iodine is all disengaged. This white vapour is produced by an acid substance, in which we have recognised the following properties: $\mathrm{Jt}$ does not dissolve iodine; it possesses a flavour similar to that of dilute sulphuric acid; it gives no precipitate with muriate of barytes, but with nitrate of silver and the oxymuriate of mercury it does (the latter precipitate of a pale red colour); and fills the atmosphere with the odour of chlorine. We purpose afterwards to examine this product.

The iodine may be disengaged at the same time with the muriatic acid, and as soon as that acid, by applying at once a boiling heat. The vapours which appear are violet, and the little muriatic acid which passes at the same time is not particularly coleured. It has been said, that at the degree of heat at which iodine can be produced, the action of the sulphuric acid tends rather to produce this body than to disengage the muriatic acid. It is true that the strongest affinity is then in favour of the muriates, and that the weakest acids, perhaps even sulphurous acid, may decompose the oxygenated iodates. The operation, whatever may be the quantity of salt, is finished in an hour at the most. The saline residue, in an experiment which had been interrupted after the disengagement of iodine, was composed in its liquid part, which was decanted warm, of super-sulphate of soda and super-sulphate of potash, besides free sulphuric acid; the remaining part, concrete when warm, consisted of muriate of potash and muriate of soda. This proves that muriatic acid had concurred in the decomposition of the oxygenated iodate of potash. It appeared singular that acidulated sulphates and free sulphuric acid had been able to exist together, and to be heated to ebullition, without being mutually decomposed. After having distingaished the different salts, we joined them to the mother-waters, and distilled the mixture to dryness. A great deal of muriatic acid passed, and there only remained neutral sulphates of potash and soda.

In a similar experiment, but which was not interrupted after the disengagement of the iodine, a great deal of muriatic acid passed at the distillation. This acid was absolutely colourless, and almost without odour. However, a good deal of muriate of iron was found in the residue, and the acid obtained had enough of concentration to fume if it had been otherwise prepared.

We made the experiment of washing with a little water the rough kelp finely pulverised. The solution contained more iodate and less of other salts than the lye made in a large quantity of 
water ; but there remained after this mode of extraction, still too much iodate in the residue to make it of advantage to follow it.

If the separation of the crystallizable salts is pushed too far, and especially if it is songht to obtain them by the recooling of the lye, nothing, as we have said before, will be preserved but a mother-water exhausted of iodate, and which will not yield the least part of iodine. It is from not finding iodine in the motherwater of a soda known to contain it, that we have thought of seeking for it in the crystallized salt.

It has been stated that, from Scotch kelp, iodate of soda as well as iodate of potash may be extracted. That kelp cannot therefore reckon amongst its salts either the sulphate or muriate of potash, which would be decomposed by the iodic acid of the iodate of soda, although there may be found in the same lye the sulphate of soda and the muriate of potash. It is impossible, however, to admit, as some authors have done, the existence of these different salts with the iodates of magnesia and of lime.

The soda with which we have experimented was of the species known in commerce under the name of common soda of Fecamp. The pieces recently broken presented in their fracture a blueish gray colour; they were besprinkled with opaque white crystalline knots; the parts which had been exposed to the contact of the air were humid, of a black colour, and contained also knots of salt. Their lye gave about a fourth of their weight in saline products, but no sub-carbonate of soda. The water of a first cold washing contained muriate of lime, and the lye obtained by boiling contained muriate of magnesia. The former muriate was no longer to be found in the lye, having been decomposed by the sulphate of soda. Common soda preserved for thirty years, and which after being damped was completely dried, did not yield less iodine than kelp newly bought. In Belgium, where considerable use is made of kelp for the fabrication of common glass, it is found to give a very good frit, and that the salts supply to that what it wants of alkali; the solution of its residue after the separation of the iodine, leaving on the filter a good deal of silex. The alkali may be considered as being partly in the state of liquid silicate; which explains how its lye in the neutral alkali saturates a certain quantity of acid.

We have tried to decompose at the outset the muriatic salts, by pouring into the lye a quantity of sulphuric acid-at first, because that method accelerated the operation-afterwards, because we found that in operating with a salt which is not very dry, or which has got moist in the air, the muriatic acid which passes, and the expulsion of which demands a greater degree of heat, is always much coloured with iodine; but we have in experiments 


\section{On the Method of extracling Iodine from Kelp.}

on a large scale dissipated in air the muriatic gas by decomposing with a slow fire the salt mixed with the sulphuric acid.

In following our process, no more need at first be incorporated than the sulphuric acid indispensable for the decomposition of muriates; and after that decomposition, the remainder of the acid may be added by spreading it uniformly over the whole mass of salt; but this precaution is not necessary when the process is followed throughout in the way which we have pointed out.

The separation of the salts may be also attempted by subjecting their mixture in a dry state to a heat capable only of liquifying the iodate of potash, and the sulphate of soda; the supernatant liquid will cover two muriates, and the sulphate of potash not melted.

We expected to have found the means of separating the iodate with the muriatic acid, when we had poured the ammoniacal liquid on the acid, which passed red at the distillation. There was not at first any mixture between the two liquids; but on coming in contact they were observed to separate, and crystallize in irregularly faced pyramids with their base turned upwards. The crystals were very numerous. We expected that by agitation they would have fallen to the bottom of the liquid, but it made them disappear; some new alkali reproduced them, and that until the ammonia was in excess;-the colour of the acid, far from disappearing, became livelier. We repeated this experiment several times, and always with the same result. We made the experiment each time with three or four ounces of liquid. Thus, in that operation which without contradiction had been the simplest and the easiest, our attempt was ursfortunately abortive.

We made an experiment on the volatility of iodine in air : five grains of crystal of that substance were placed in the morning on the reverse of a cup;-towards evening a great part had been already dissipated ; and next morning the whole had disappeared. The temperature of the apartment varied from $8^{\circ}$ to $10^{\circ} \mathrm{R}$., and the odour of chlorine continued for several days.

Two grains of iodine placed in the palm of the hand, dissipated in less than twelve minutes; the spot remained much longer. It was when at another time making the same experiment, with the view of ascertaining the identity of the odour of iodine with that of chlorine, that we discovered the property which the former substance has of colouring starch blue. A napkin passed through starch, with which we wiped our hands, was covered with large blue spots, which by little and little became violet, and ultimately disappeared. Since then we have employed as a reagent for iodine small strips of cloth steeped in starch water. 
When the existence of iodic acid is suspected in a salt, add to a solution of this salt a little of the water of chlorine; a reddishbrown colour will immediately appear, from the iodine dissolving in the muriate ; by adding a little starch-water; a deep blue colour will be obtained : if an excess of chlorine is added, the redbrown colour disappears, and the starch-water is no longer coloured blue; - the water thus coloured, loses itself all its colour by the addition of a little water of chlorine. The very marked development of the muriatic acid denotes that the oxygen passes to the iodine. When in these experiments the iodine is in a greater quantity than the starch, the colour is of a deep cærulean blue;-if, on the contrary, the starch is in excess, the colour is of a brownish black; and at the point of saturation it is a beautiful violet. A particle of water containing iodate, dropped on paper which has been starched and placed above a bottle containing chlorine, produces instantly a beautiful blue spot.

Such have been hitherto the results of our experiments, which we purpose yet to extend as far as the object will allow.

\section{Present State of the Ruins of Babylon. By Captain EDWARD FREDERICK*.}

$\mathbf{T}$ H E interesting descriptions given bỳ Captain F. will doubtless have great future importance in guiding travellers to the site of these famous ruins of the East, the way to which he shows very accurately. He observes, " that the ruins of the mounds lie on the left, a short distance off the direct road from Hillah; and a traveller merely sees Belus's tower as he rides along, and must turn out of his way if he wishes to examine it, which will occupy a longer time than the travellers generally have leisure for, as appears from their own acknowledgements, not to notice their dread of being surprised by the wandering Arabs.

"As to the other travellers who have visited this celebrated spot, it would be carrying complaisance too far to place implicit confidence on their relations, as they appear merely to have passed over the ground, and sometimes not even to know that they were amidst the ruins, until their guides told them it was Babel they were riding over. They of course had no time to examine the heaps of rubbish. Other travellers visited only one bank of the Euphrates, not caring to risk meeting with the Arabs while gratifying their curiosity on the other. From Belus's tower (which is four miles from Hiltah in a direct line) there are no more

* From the Transactions of the Literary Society of Bombay. 